\title{
Course of Antibody Response in Lyme Borreliosis Patients before and after Therapy
}

\author{
Elisabeth Aberer ${ }^{1}$ and Gerold Schwantzer ${ }^{2}$ \\ ${ }^{1}$ Department of Dermatology and Venereology, Medical University of Graz, Auenbruggerplatz 8, 8036 Graz, Austria \\ ${ }^{2}$ Institute for Medical Informatics, Statistics and Documentation, Graz, Medical University of Graz, 8036 Graz, Austria
}

Correspondence should be addressed to Elisabeth Aberer, elisabeth.aberer@medunigraz.at

Received 27 September 2011; Accepted 27 October 2011

Academic Editors: A. Clayton and S. Devi

Copyright (C) 2012 E. Aberer and G. Schwantzer. This is an open access article distributed under the Creative Commons Attribution License, which permits unrestricted use, distribution, and reproduction in any medium, provided the original work is properly cited.

\begin{abstract}
The early immune response (IR) in European Lyme borreliosis patients has not yet been studied in detail. The aim of the study was to analyse retrospectively the antibody development in 61 erythema migrans (EMs) patients depending on the duration of infection from tick bite by using a whole-cell lysate $B$. garinii immunoblot. The evolution of antibodies proved to be undulatory in untreated patients with two peaks for IgM at weeks 5 and 9 and for IgG at weeks 4 and 8 . The analysis of IR courses after therapy identified patients constantly seropositive or seronegative and patients with repeated seroconversions with a switch, disappearance, or reappearance of anti- $23 \mathrm{kD}$ or anti-39 kD antibodies during the one-year period. We suggest that the antibody production in EM patients may be missed due to an undulatory IR. This phenomenon might be an as yet insufficiently researched aspect in Lyme borreliosis.
\end{abstract}

\section{Introduction}

Serological testing aids in the diagnosis of Lyme borreliosis (LB). IgM antibodies develop during the first 3 weeks after infection followed by IgG antibodies after 4-6 weeks [1]. However, to our knowledge, the early immune response (IR) in European patients has not yet been studied in detail.

In routine clinical practice, we observed patients whose IR switched from positive to negative and vice versa in the course of time. The aim of this study was to analyse the development and the course of the IR in European erythema migrans (EMs) patients with known duration of disease from tick bite and to follow up their antibody profile during a 12month period after treatment.

\section{Patients, Materials, and Methods}

One hundred and two patients with EM were enrolled for clinical and serological diagnosis. Their mean age was 50 (SD16) years. Forty-seven patients were male and 55 female. The mean duration of EM was 23 (1-210) days. Eightyseven patients had EM without and 15 patients EM with extracutaneous symptoms. As sixty-one (28 males and 33 females aged between 22 and 78 years) remembered their tick bite, the onset of their infection was known and only these patients were included in the study. These patients received antibiotic treatment with phenoxymethylpenicillin 1500000 IE threetimes a day, 26 patients for 14 days and 35 patients for 20 days. No statistical difference in the clinical outcome of the differently treated groups was observed during the 12-month observation period as described in a previous study [2].

Blood samples were obtained from all 61 patients for serological testing at the initial visit before therapy and then thereafter at 3 weeks after first blood withdrawal. Sera were available from 46 patients 3 months after therapy, from 52 after 6 months, and from 53 after 12 months (Table 1).

Patients' sera that had been stored at $-20^{\circ} \mathrm{C}$ were retested at the same time and antibody response was measured by a whole-cell lysate immunoblot (IB) according to the manufacturer's instruction (Borrelia garinii, MRL Diagnostics, Cypress, CA). Interpretation of the IB bands was performed by a single technician. Criteria for a positive IB, as indicated in the manufacturer's manual, were 1 of 2 bands 
TABLE 1: Number of investigated patients' sera.

\begin{tabular}{lc}
\hline No. of sera & Time of enrolment \\
\hline 102 & Enrolled EM patients \\
61 & Tick bite known \\
61 & Before treatment \\
61 & After treatment \\
46 & 3 months after treatment \\
52 & 6 months after treatment \\
53 & 12 months after treatment \\
\hline
\end{tabular}

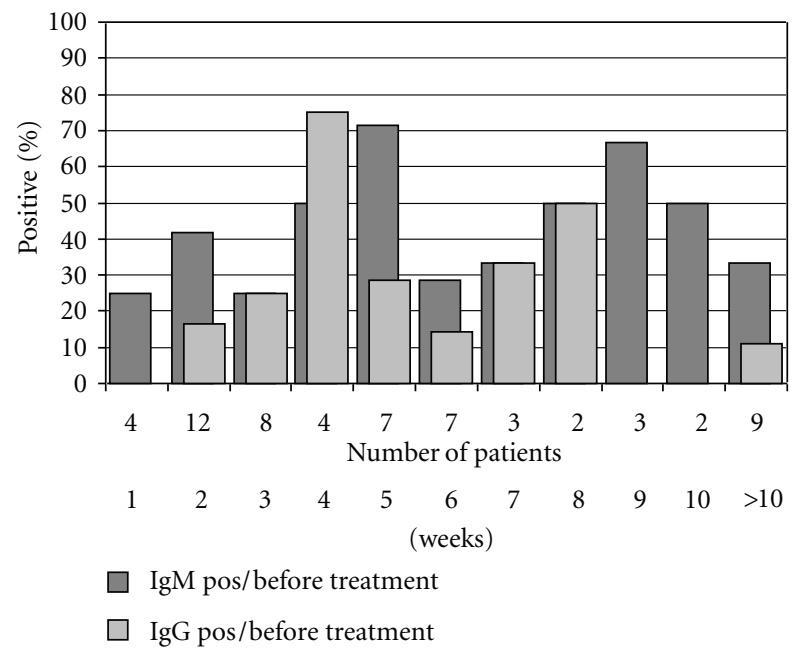

FIGURE 1: Development of immune response from tick bite (weeks).

(23 and $39 \mathrm{kD}$ ) for $\operatorname{IgM}$ and 4 of 7 bands $(21,23,37,39$, 41,45 , and $93 \mathrm{kD}$ ) for IgG. Data obtained were entered in a computerized database and graphically displayed.

\section{Results and Discussion}

3.1. Development of Immune Response. Twenty-five of 61 (41\%) sera showed IgM antibodies in IB before therapy. The IR started in week 1 after tick bite and peaked in week 5 , when 5 of the 7 tested sera (71\%) reacted positively (Figure 1). In the following weeks there was a wavelike decrease of seropositive reactions. Furthermore, from week 8 there was a second increase in seropositivity peaking at week 9. IgG antibodies were positive in $13 / 60(21 \%)$ patient sera (one serum could not be analysed because of a smear). The IR started from week 2 and peaked at week 4, where 75\% (3 of the 4 tested sera) reacted positively (Figure 1). Similar to the IgM antibody trend, the IgG IR decreased in the following weeks. However, there was a second increase of IgG IR that peaked in week 8 .

The development of the IR to an infection with $B$. burgdorferi $(B b)$ s.s. in EM patients from the United States was studied by Aguero-Rosenfeld and showed that $43 \%$ of the patients had positive IgM antibodies before therapy [3]. Due to the heterogeneity of borrelia strains in Europe, differences between IR on the two continents are expected

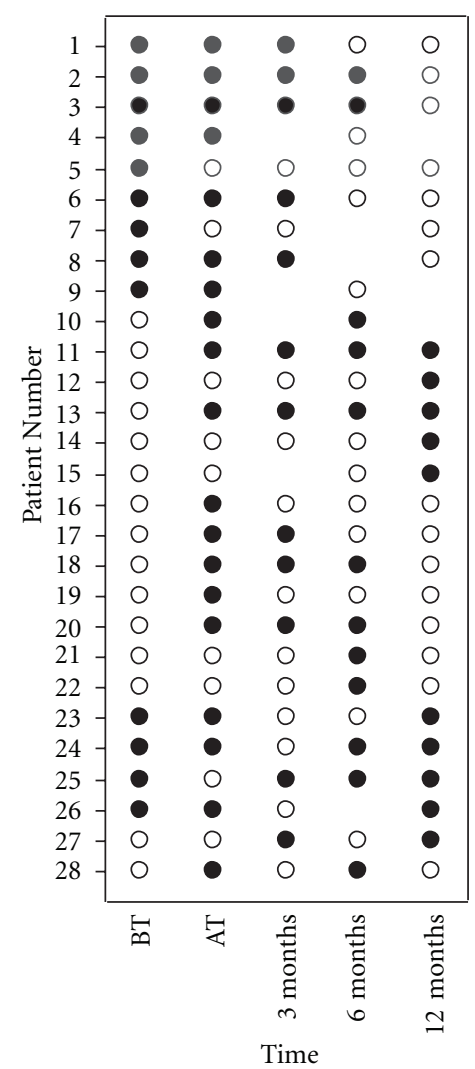

Figure 2: IgM antibody response in immunoblot before and up to 12 months after therapy. Filled circles: positive $\operatorname{IgM}$ antibody response; empty circles: negative IgM antibody response; blank spaces: no serum available. BT: before therapy; AT: after therapy; $3,6,12$ months after start of therapy.

since the $B b$ s.s. species provokes a stronger immune reaction than the dominant European species B. afzelii. Patients with culture-confirmed B. burgdorferi s.s. erythemas from the USA were more often seropositive $(35.3 \%)$ at presentation compared with $22.4 \%$ culture-confirmed B. afzelii erythemas [4]. In a another European study with recombinant ELISA, German Lyme borreliosis patients yielded positive IgG antibodies in $22 \%$ and IgM antibodies in $61.5 \%$ for phase I of LB [5].

Both the IgM and IgG IR showed an undulatory distribution with antibodies coming and going in untreated patients over weeks. A similar periodicity is known in other bacterial infections, like relapsing fever with recurrent bacteraemias [6]. Syphilis can be reactivated by different conditions like HIV infection, showing seroconversion of the unspecific VDRL [7].

3.2. Course of Immune Response after Treatment. With respect to the course of IR after therapy, 21 of 61 (34\%) patients did not show IgM seroconversion (constantly negative), whereas $12(20 \%)$ were constantly positive. In the remaining 28 patients, different kinds of IgM seroconversion occurred. Nine patients (15\%) (Figure 2, patients 1-9) seroconverted from positive to negative and $6(11 \%)$ (Figure 2, patients 


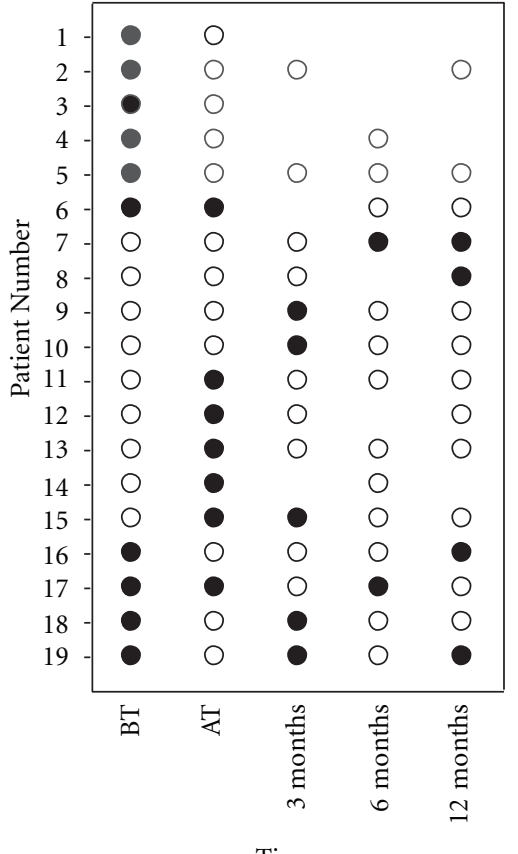

Time

Figure 3: IgG antibody response in immunoblot before and up to 12 months after therapy. Filled circles: positive IgG antibody response; empty circles: negative IgG antibody response; blank spaces: no serum available. BT: before therapy; AT: after therapy; $3,6,12$ months after start of therapy.

10-15) from negative to positive during the observation period. Seven seronegative patients (11\%) (Figure 2, patients 1622) seroconverted to positive and than back to seronegative during the 12 months. Six patients (10\%) (Figure 2, patients 23-28) showed repeated seroconversions of IgM antibodies that presented as a switch of anti- $23 \mathrm{kD}$ to anti-39kD antibodies and vice versa or the dis- or reappearance of either anti- $23 \mathrm{kD}$ or anti-39 $\mathrm{kD}$ antibodies.

Thirty-eight of the 60 patients $(63 \%)$ were constantly IgG negative and 3 patients (5\%) constantly positive in IB. Nineteen patients seroconverted within the observation period. Their data are presented in Figure 3. Six patients (10\%) (patients 1-6) seroconverted from initially positive to negative and $2(3 \%)$ (Figure 3, patients 7 and 8 ) from negative to positive. Seven patients (12\%) (Figure 3, patient 9-15) who were primarily negative seroconverted to positive and then back to seronegative during the observation period.

Four patients (7\%) (Figure 3, patients 16-19) showed repeated seroconversion with at least 2 changes. The seroconversion itself presented as a loss of detectable antibodies from a previous maximum of up to 5 bands $(23,37,39,41,45$, and $93 \mathrm{kD}$ ) to 1 band.

When observed closely, none of the patients with a repeated seroconversion had any features that could distinguish them from the other patients with either persistent antibodies or seronegative individuals. There was also no relation between IgM and IgG seroconversion. Clinically, no correlation to the duration of treatment or to the presence of extracutaneous signs could be drawn.

A recent survey on the development of the IR, measured by ELISA, in Austrian EM patients over a minimum of 1 year after treatment showed 3 distinct courses: persistent positive, persistent negative, and positive to negative patients [8]. In this study, there were also patients with a change from negative to positive antibodies (3\% IgM, $4 \% \operatorname{IgG})$, but this was not attributed to the original EM as seroconversion occurred after a median of 350 (for $\operatorname{IgM}$ ) and 349 days (for IgG) after EM. In our study, IB was found to be more sensitive than ELISA; therefore only IB results were analyzed and the IR could be studied in more detail.

In our patients, the appearance of IgM antibodies only detected after 6 months (patients 21 and 22) and $\operatorname{IgG}$ antibodies only at 3 months (patients 9 and 10) cannot be related to the previous infection. Moreover, an isolated $\operatorname{IgM}$ seroreaction has been observed to be unspecific $[9,10]$. Isolated positive serum IgM titers were seen in about $20 \%$ of children with a febrile illness, enterovirus meningitis, or headache [9]. In another article $2,6 \%$ of sera submitted for B. burgdorferi serology expressed unspecific anti-p41 IgM antibodies by ELISA. Confirmation test with IB showed that some sera also reacted with $\mathrm{p} 39$ or OspC antigen. No conclusive evidence for borrelia infection could be drawn [10].

The first appearance of antibodies after 6 and 12 months in our study (patients 14 and 15 for IgM, and 7 and 8 for IgG) might indicate a new infection in these patients.

Although the undulatory character of the IR before therapy in our patients could not be determined in every single patient, the findings after treatment might reflect a similar situation also in untreated patients before therapy. So, we suggest that a single serological finding is a snap shot and gives evidence of an infection. On the other hand, the true infection might be missed by negative IR, as might be the case in the $\sim 40 \%$ seronegative EM patients.

Serological findings do not distinguish between active and previous disease. Borrelia DNA can persist in urine for even 1 year after treatment [11], and antibodies to $B b$ may persist for up to 20 years after appropriate therapy [12]. Bearing in mind the characteristics of cyclic patterns in other bacterial infections, the undulatory IR noted in our study may be an as yet insufficiently researched aspect in Lyme borreliosis.

\section{Conflict of Interests}

The authors declare that there is no conflict of interests.

\section{Acknowledgments}

The authors greatly acknowledge Jasmina Custovic, M.D., for collecting and analysing the data which were also partly shown in her thesis (development of the immune response in erythema migrans with special significance of the p18 antigen) and Mrs. Ingrid Krainberger for her excellent laboratory support during the study. 


\section{References}

[1] B. Wilske, "Serodiagnosis of lyme borreliosis," Zeitschrift fur Hautkrankheiten, vol. 63, no. 6, pp. 511-514, 1988.

[2] E. Aberer, P. Kahofer, B. Binder, T. Kinaciyan, H. Schauperl, and A. Berghold, "Comparison of a two- or three-week regimen and a review of treatment of erythema migrans with phenoxymethylpenicillin," Dermatology, vol. 212, no. 2, pp. 160-167, 2006.

[3] M. E. Aguero-Rosenfeld, J. Nowakowski, S. Bittker, D. Cooper, R. B. Nadelman, and G. P. Wormser, "Evolution of the serologic response to Borrelia burgdorferi in treated patients with culture-confirmed erythema migrans," Journal of Clinical Microbiology, vol. 34, no. 1, pp. 1-9, 1996.

[4] F. Strle, R. B. Nadelman, J. Cimperman et al., "Comparison of culture-confirmed erythema migrans caused by Borrelia burgdorferi sensu stricto in New York State and by Borrelia afzelii in Slovenia," Annals of Internal Medicine, vol. 130, no. 1, pp. 32-36, 1999.

[5] K. P. Hunfeld, M. Ernst, P. Zachary, B. Jaulhac, H. H. Sonneborn, and V. Brade, "Development and laboratory evaluation of a new recombinant ELISA for the serodiagnosis of Lyme disease," Wiener Klinische Wochenschrift, vol. 114, no. 13-14, pp. 580-585, 2002.

[6] C. Larsson, M. Andersson, J. Pelkonen, B. P. Guo, A. Nordstrand, and S. Bergström, "Persistent brain infection and disease reactivation in relapsing fever borreliosis," Microbes and Infection, vol. 8, no. 8, pp. 2213-2219, 2006.

[7] A. McMillan, H. Young, and J. F. Peutherer, "Influence of human immunodeficiency virus infection on treponemal serology, in patients who have been treated for syphilis," Journal of Infection, vol. 21, no. 1, pp. 95-103, 1990.

[8] M. Glatz, M. Golestani, H. Kerl, and R. R. Müllegger, "Clinical relevance of different IgG and IgM serum antibody responses to Borrelia burgdorferi after antibiotic therapy for erythema migrans: long-term follow-up study of 113 patients," Archives of Dermatology, vol. 142, no. 7, pp. 862-868, 2006.

[9] R. Bennet, V. Lindgren, and B. Zweygberg Wirgart, "Borrelia antibodies in children evaluated for Lyme neuroborreliosis," Infection, vol. 36, no. 5, pp. 463-466, 2008.

[10] E. Ulvestad, A. Kanestrøm, L. J. Sønsteby et al., "Diagnostic and biological significance of anti-p41 IgM antibodies against Borrelia burgdorferi," Scandinavian Journal of Immunology, vol. 53, no. 4, pp. 416-421, 2001.

[11] E. Aberer, A. R. Bergmann, A. M. Derler, and B. Schmidt, "Course of Borrelia burgdorferi DNA shedding in urine after treatment," Acta Dermato-Venereologica, vol. 87, no. 1, pp. 3942, 2007.

[12] R. A. Kalish, G. McHugh, J. Granquist, B. Shea, R. Ruthazer, and A. C. Steere, "Persistence of immunoglobulin M or immunoglobulin $\mathrm{G}$ antibody responses to Borrelia burgdorferi 1020 years after active Lyme disease," Clinical Infectious Diseases, vol. 33 , no. 6 , pp. 780-785, 2001. 


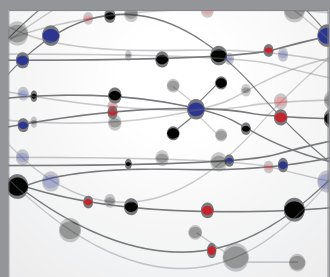

The Scientific World Journal
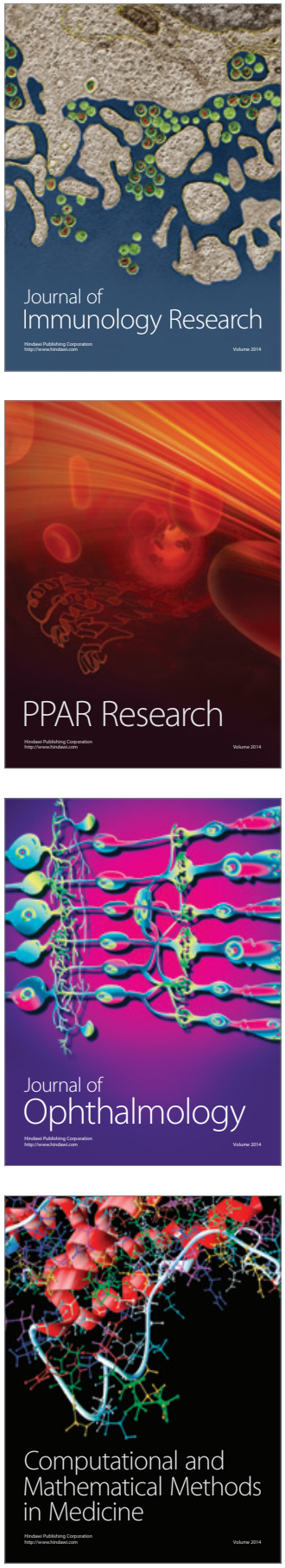

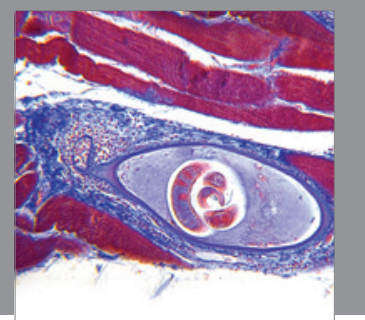

Gastroenterology

Research and Practice
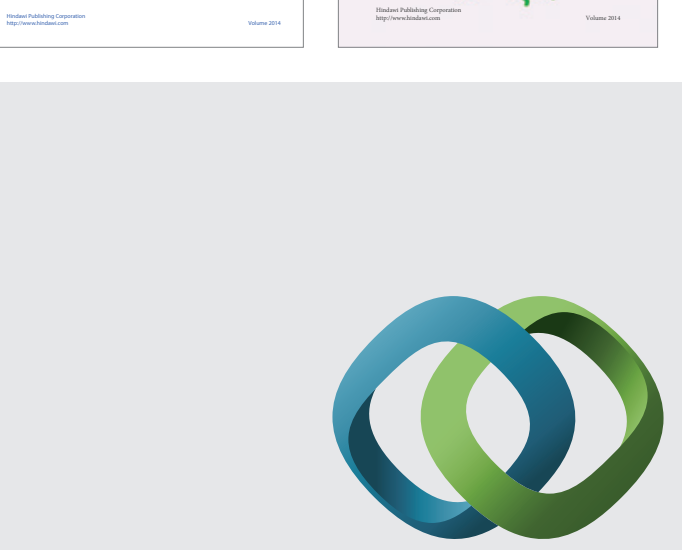

\section{Hindawi}

Submit your manuscripts at

http://www.hindawi.com
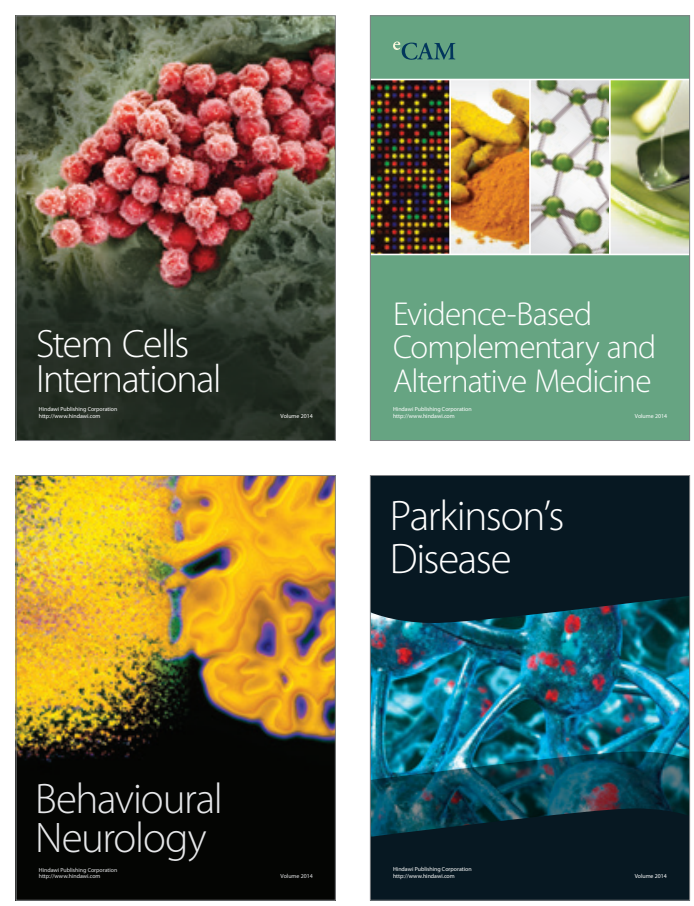

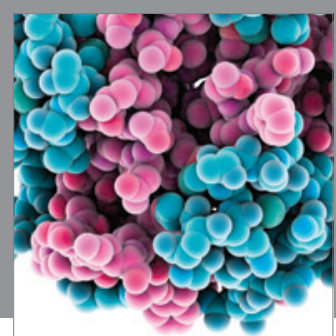

Journal of
Diabetes Research

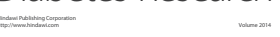

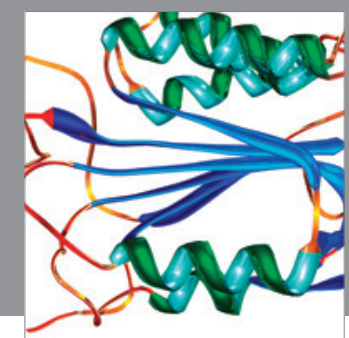

Disease Markers
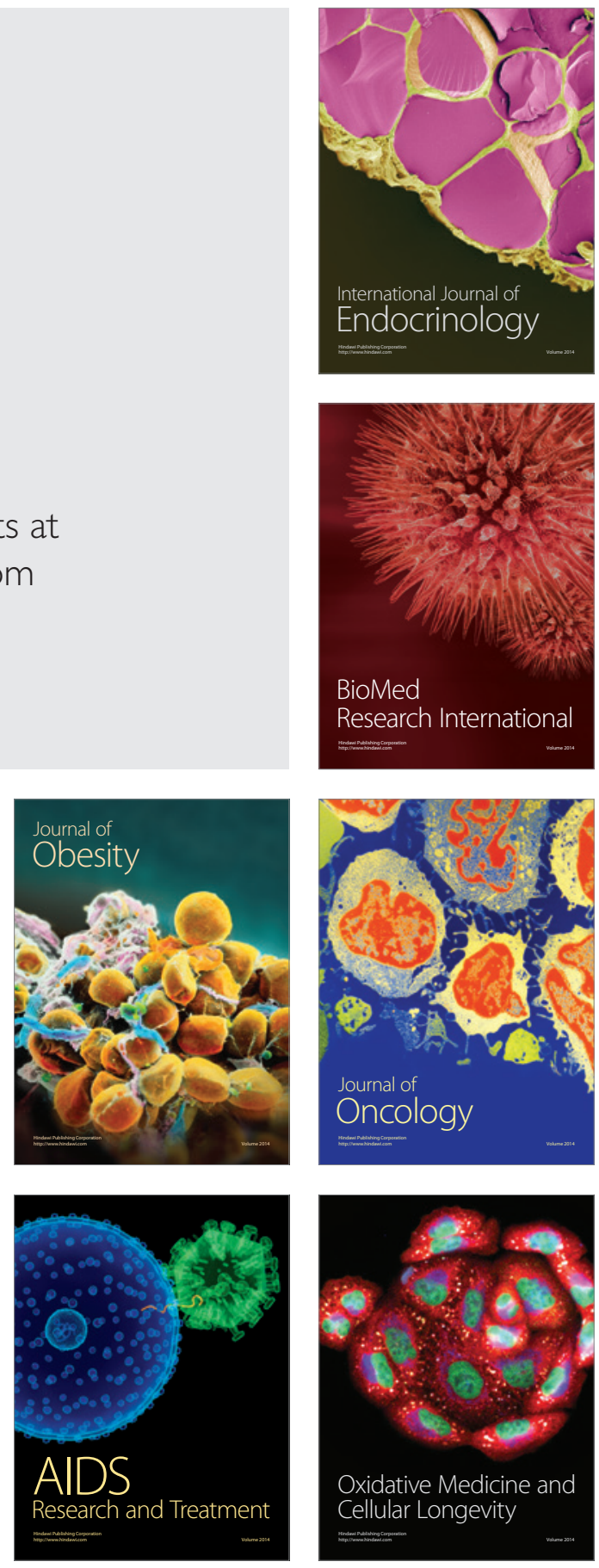